\title{
A Novel Approach to Future Horizon of Top Seven Biomedical Research Topics to Watch in 2017: Alzheimer's, Ebola, Hypersomnia, Human Immunodeficiency Virus (HIV), Tuberculosis (TB), Microbiome/Antibiotic Resistance and Endovascular Stroke
}

\section{Alireza Heidari \\ Faculty of Chemistry, California South University, 14731 Comet St. Irvine, CA 92604, USA}

*Corresponding author: Alireza Heidari, Faculty of Chemistry, California South University (CSU), 14731 Comet St. Irvine, CA 92604, USA, Tel: +1-775-410-4974; Email: Scholar.Researcher.Scientist@gmail.com

Received date: May 15, 2017; Accepted date: May 25, 2017; Published date: June 15, 2017

Copyright: (c) 2017 Alireza $\mathrm{H}$. This is an open-access article distributed under the terms of the Creative Commons Attribution License, which permits unrestricted use, distribution and reproduction in any medium, provided the original author and source are credited.

\section{Editor's Note}

It was British scientist Heinz Wolff, who coined the term 'Bioengineering' for the first time in the year 1954. However, first ever project on 'Bioengineering and Biomedical' field was undertaken at Mississippi State University in the year 1967. With the progress of time, bioengineered crops and various other biomedical applications enriched the scientific knowledge towards social welfare. In present day's understanding on Bioengineering and Biomedical Science, implies an interdisciplinary study that synchronizes engineering and design technologies to resolve biological problems. Both these fields strive to develop new technologies, interventions, and by extension, knowledge which culminates in betterment of human life.

The Journal of Bioengineering \& Biomedical Science provides a platform for dissemination of knowledge relating to all sub-specialties of Bioengineering and Biomedical Science such as biomedical equipment, biometrics, bioprocessing, bioreactors, biosensors, etc. The current issue of the Journal of Bioengineering \& Biomedical Science presents some interesting findings and advancements in the field. Bassey et al. [1], developed a mathematical model for scoring the optimal benefits of chemotherapy with respect to the patients' CD4+ T-cell counts in response to Reverse Transcriptase Inhibitor (RTI) treatment. Huang et al. [2], postulated that, the positive effects of Pulsed Electromagnetic Fields (PEMF) on bone structure can be attributed to its regulation of bone resorption, and elevation of the Bone Mineral Density (BMD), through the intervention of the RANK and CN/NFAT pathways. Xiaobing Yu et al. [3], found that FERA, IERA and VERA can be used as a diagnostic reference for Anterior Cruciate Ligament (ACL) injury. Ahmed et al. [4], using Electroencephalography (EEG) for predicting seizures; they achieved high prediction efficiency (96.24\%) in minimum time (54.01 min) with an extremely low false alarm rate $(0.10526 / \mathrm{h})$. Waseem et al. [5] observed that the elastic modulus of the bovine hip joint deteriorated in both the longitudinal and transvers directions with increasing age. Sosaku et al. [6], developed lipid vesicles in nanometer range which exhibit high entrapment efficiency with respect to hydrophilic molecules. David et al. [7], identified that, organic Carbon can be harvested from abattoir wastewater to perform anoxic denitrification.

The treatment of Human Immunodeficiency Virus (HIV), which frequently transmutes into Acquired Immune Deficiency Syndrome (AIDS) and its auxiliary diseases, remains a labyrinthine task. Newer combinations of HIV such as HIV-hepatitis C, HIV-parasitoid pathogen, and HIV-tuberculosis are continually evolving. Ten percent of the HIV infections worldwide involve recombinant viruses: It has also been reported that, the first confirmed report of HIV dual infection in humans occurred in 2002; HIV triple infection has also been reported. As of date, suppression and prevention have been found to be the bulwark of HIV management. Effective management of dual HIV infections involves chemotherapy, a process that involves clinical modelling. Bassey et al. [1], developed a 4-dimensional mathematical model that scored for the optimal benefits of methodical administration of chemotherapy, in terms of the patients' CD4+ T-cell counts in response to Reverse Transcriptase Inhibitor (RTI) treatment. The analyses using this model revealed that, virus management is a direct function of compliance and standardization of the chemotherapy regimen. Furthermore, high intensity drug dosage at the onset of treatment results in lower costs incurred by the patient. The model further recommends incorporation of multiple immunotherapies for bypassing early drug resistance.

Pulsed Electromagnetic Fields (PEMF) may affect osteogenesis and by extension, be used as a treatment for osteoporosis. Low frequency, single pulse electromagnetic fields have been observed to suppress trabecular bone loss and restore trabecular bone structure and in rat models of osteoporosis. Huang et al. [2], investigated the transcript levels of biomolecules in involved in osteogenesis and bone resorption to account for the beneficial effects mediated by PEMF. Towards this, the authors analysed the expression of CAII, NFAT2, RANK, and VATPase transcripts in rat models of osteoporosis. All mRNA levels were analysed in response to ovariectomy with PEMF stimulation (PEMF+OVX) Versus Ovariectomy (OVX) alone and SHAM. It was observed that the PEMF treatment group exhibited significantly lower expression of CAII, NFAT2, RANK, and V-ATPase than the OVX group. At the same time, with respect to SHAM, PEMF treatment group exhibited significantly higher expression of CAII, RANK, and $\mathrm{V}$-ATPase. These data suggest that PEMF controls bone resorption and elevates the Bone Mineral Density (BMD), through the intervention of the RANK and CN/NFAT pathways. Simultaneously, PEMF may also promote the BMD upregulation of NFAT.

Xiaobing Yu et al. [3], evaluated the characteristic features of gait in Anterior Cruciate Ligament (ACL) injury with respect to the healthy control group using a three-dimensional gait capturing system (MoCap system: DVMC ${ }^{\circledR}-8820$ ). The authors studied the Internal and External Rotation Angle (IERA), the knee Flexion Extension Rotation Angle (FERA), and Varus Eversion Rotation Angle (VERA) during the swing and stance phases of the gait cycle. In the ACL group, the knee flexion angle, the tibial external rotation and the varus reached the maxima during the swing phase. While in the stance phase, the tibial internal rotation, the varus angles, and the extension reached a 
Citation: Alireza Heidari (2017) A Novel Approach to Future Horizon of Top Seven Biomedical Research Topics to Watch in 2017: Alzheimer's, Ebola, Hypersomnia, Human Immunodeficiency Virus (HIV), Tuberculosis (TB), Microbiome/Antibiotic Resistance and Endovascular Stroke. J Bioengineer \& Biomedical Sci 7: e127. doi:10.4172/2155-9538.1000e127

Page 2 of 2

maxima. The main difference lie in the fact that the FERA, IERA and VERA varied within a narrow range in the healthy control group whereas, they significantly varied in ACL group. As the differences between the two groups are so pronounced, FERA, IERA and VERA can be used as a diagnostic reference for ACL injuries. EEG signal processing has numerous applications in medicine such as seizure prediction and detection, but seizure prediction needs further refining. Ahmed et al. [4], worked on refining the Efficacy of Electroencephalography (EEG) for predicting seizures. The work involved EEG channel selection based on the probability distribution of EEG signals. By incorporating this strategy, the authors achieved high prediction efficiency (96.24\%) in minimum time (54.01 min) with an extremely low false alarm rate $(0.10526 / \mathrm{h})$. As this method does not have a high computational load, it can be implemented in future studies of seizures. The decline in the mechanical properties of bone associated with aging is the underlying cause of bone fracture in elderly people; the pivotal feature associated with decline in mechanical property of bone is the elastic modulus or Young's Modulus, which gets deteriorated in aging bones. The mechanism behind this age dependent deterioration of the Young's Modulus is unknown. Waseem et al. [5] presented a study based on tensile testing experiments conducted on the bovine hip joint bone. The study was performed in both longitudinal and transverse directions for investigating the effect of age on the elastic modulus of the bone. The authors observed that the elastic modulus deteriorated in both the longitudinal and transvers directions in an age dependent manner. Further, the deterioration was higher in transverse direction as compared to the longitudinal direction. Extensive research has been conducted on liposomes mediated delivery of biomolecules and bioactive compounds. Multiple attempts are being made to enhance nano- and micro- dispersion properties, especially with respect to the stability, entrapment efficiency, and size. Liposomal entrapment of hydrophilic molecules is problematic as both entrapment efficiency and stability are hampered. Sosaku et al. [6], developed lipid vesicles in nanometer range exhibiting high entrapment efficiency with respect to hydrophilic molecules. For vesicle preparation, the authors employed multiple emulsion method whose workflow includes processes such as ultrasonic emulsification homogenization, MC emulsification, and evaporation based removal of organic solvent. The average size of these vesicles was $182(\mathrm{~nm})$ and; the vesicle size was comparable to the droplet size of the primary emulsion. High entrapment values were achieved for hydrophilic molecules such as Calcein $(89.3 \pm 4.2 \%)$ and for 5-fluorouracil $(41.1 \pm 3.3 \%)$. Thus, the multiple emulsion method can be employed across industries.Nitrate-rich effluents adversely affect the environment and public health. The elimination of nitrates requires a denitrification step which is dependent on the presence of organic Carbon. David et al. [7], evaluated the potential of utilizing the organic Carbon present in effluents for performing denitrification. Towards this, wastewater from abattoirs was used. The authors observed that, significant denitrification was achieved using $10 \%$ of anaerobically-treated abattoir wastewater. Therefore, it is possible to harvest enough organic carbon from effluents of abattoirs for performing anoxic denitrification; this rate however, is a function of the organic Carbon load.

\section{References}

1. Bassey E, Kimbir, Anande RK, Andreyevich KL (2016) On Optimal Control Model for the Treatment of Dual HIV-Parasitoid Pathogen Infection. J Bioengineer \& Biomedical Sci 6: 212.

2. Huang H, Chen J, He JQ, Fu JF (2017) Study on Pulsed Electromagnetic Fields Regulate the mRNA Expression of V-ATPase, CAII, NFAT2 and RANK in an Ovariectomized Rat Model of Osteoporosis Mechanism Analysis. J Bioengineer \& Biomedical Sci 7: 217.

3. Xiaobing Yu, Chunhui Li, Yupeng Liu, Xiaojun Ma, Weiming W (2017) Three- Dimensional Gait Analysis of Anterior Cruciate Ligament Before and After Injury. J Bioengineer \& Biomedical Sci 7: 215.

4. Ahmed S, El-Khobby H, Mahmoud A, El-Samie AFE (2017) Enhanced Technique via Filters for Seizure Prediction. J Bioengineer \& Biomedical Sci $7: 218$.

5. Waseem UR (2017) Effect of Age on the Elastic Modulus of Bone. J Bioengineer \& Biomedical Sci 7: 219.

6. Sosaku I, Yuya O, Emmanuel CO, Takashi KB, Yasuyuki M, et al. (2016) Preparation of Lipid Vesicles having Suitable Size for Drug Delivery with High Entrapment of Hydrophilic Molecules Using Multiple Emulsions. J Bioengineer \& Biomedical Sci 7: 213.

7. David NM, Eliud Nyaga MN, George O, Frank K, John O, et al. (2017) Evaluation of Organic Carbon from Anaerobic Sequencing Batch Reactor Effluent as a Carbon Source for Denitrification. J Bioengineer \& Biomedical Sci 7: 214. 